ANDRÉ GIDE 


\title{
CENTRE CULTUREL INTERNATIONAL DE CERISY-LA-SALLE
}

Quelques volumes parus:

Entretiens sur les notions de GENESE ET DE STRUCTURE

(Juillet-Aoat I959)

Sous la direction de MAURICE DE GANDILLAC, LUCIEN GOLDMAN BT JBAN PIAGBT 1965. 358 p. 38 F./f 27.50

\author{
Entretiens sur \\ MARCEL PROUST \\ (Juillet 1962) \\ Sous la direction de \\ GBORgES CATTAUI ET PHILIP KOLB \\ I966. 285 p. 29 F./f 20.50 \\ Entretiens sur \\ L'HOMME ET LE DIABLE \\ (Juillet-Aoat 1964) \\ Sous la direction de \\ MAX MILNBR \\ I966. 359 p., 7 fig. 29 F./f 20.50 \\ Sous presse: \\ Entretiens sur \\ L'ART ET LA PSYCHANALYSE \\ Sous la direction de \\ DR ANDRÉ BERGE, DR ANNE CLANCIER ET PAUL RICOBUR
}




\section{Entretiens sur}

\section{ANDRÉ GIDE}

sous la direction de

MARCEL ARLAND

et

JEAN MOUTON

PARIS - MOUTON \& CO - LA HAYE

MCMLXVII 
LES ENTRETIENS SUR ANDRE GIDE

ONT EU LIEU DU 6 AU I 4 SEPTEMBRE 1964

SOUS LA DIRECTION DE

MARCEL ARLANDET JEAN MOUTON

avec la collaboration de

Auguste Angles, Yves Clogenson, Grorges-Paul Collet, Klara Fassbinder, Gabriel Germain, Alain Girard, Anne Heurgon-Desjardins, Hassan Honarmandi, Reinhard Kuhn, G. W. Ireland, Claude Martin, Margaret Main, Patrick Pollard, Henri Rambaud et Maurice Rurunad

et la participation de

Gaston Bafchtold, André Berge, Andrá Berne-Joffroy, Jacquis de Bourbon-Busset, Grorges Charaire, Marchllb Charpentirr, Arlettr Clément, Paula Crespin, Michar Decaudin, Madeleine Dengeri, Pierre Desvignes, Georges Dufouy, Jean follatn, Maurice de Gandillac, Henrt Gouhizr, Michre lioure, Clara Malraux, Albert Mammi, Jacqubline Moulin, Domintqub Noguiz, Claude Ollier, Margaret Pilcher, Dominiqub Ponneau, Jean Rucardou, Marting de Rougbmont, Micher Tamllbfer, Anne-Marib Terracini et Andréb-Pierre Viénot.

(1) I967, Mouton \& Co, Paris-La Haye

Printed in the Netherlands 